\title{
OLIGONUCLEOTIDE ANION ADDUCT FORMATION USING NEGATIVE ION ELECTROSPRAY ION MOBILITY MASS SPECTROMETRY
}

\section{J. Michael Sutton ${ }^{1}$, Noha M. El Zahar ${ }^{1,2}$ \& Michael G. Bartlett ${ }^{1}$}

${ }^{1}$ Department of Pharmaceutical and Biomedical Sciences, The University of Georgia College of Pharmacy, 250 W. Green Street, Athens, GA 30602-2352, USA

2 Department of Pharmaceutical Analytical Chemistry, Faculty of Pharmacy, Ain Shams University, Organization of African Unity Street, Abassia, Cairo (11566), Egypt

${ }^{*}$ Corresponding author. E-mail address: mgbart@uga.edu (M.G. Bartlett).

Supplementary Table 1: Structures were drawn using MarvinSketch: MarvinSketch was used for drawing, displaying and characterizing chemical structures, substructures and reactions, Marvin 17.27.0, ChemAxon (https://www.chemaxon.com). Gas Phase Basicity values obtained from NIST webbook [36]. Atomic Radii obtained from a database of ionic radii [61].

\begin{tabular}{|l|l|l|l|}
\hline & Chloride & Bromide & lodide \\
\hline \multicolumn{1}{|c|}{ Salt } & $\mathrm{C} \Gamma$ & \multicolumn{1}{|c|}{$\mathrm{Br}^{-}$} & $\mathrm{r}^{-}$ \\
\hline Anion & N/A & $318.21 \pm$ & 309.20 \\
GPB & $1.1^{*}$ & \pm \\
(kcal/mol) & & 1.96 & 2.2 \\
\hline $\begin{array}{l}\text { Atomic } \\
\text { Radii } \\
(\AA)\end{array}$ & 1.81 & & $0.20^{*}$ \\
\hline
\end{tabular}


Supplemental Figure 1: Mass Spectra of additional anionic salts sprayed with 10uM Oligo 1 sprayed with $2 \mathrm{mM} \mathrm{NFTB/15mM} \mathrm{OA} \mathrm{in} \mathrm{50/50} \mathrm{water/methanol} \mathrm{with} 75 \mathrm{uM}$ Ammonium Salt

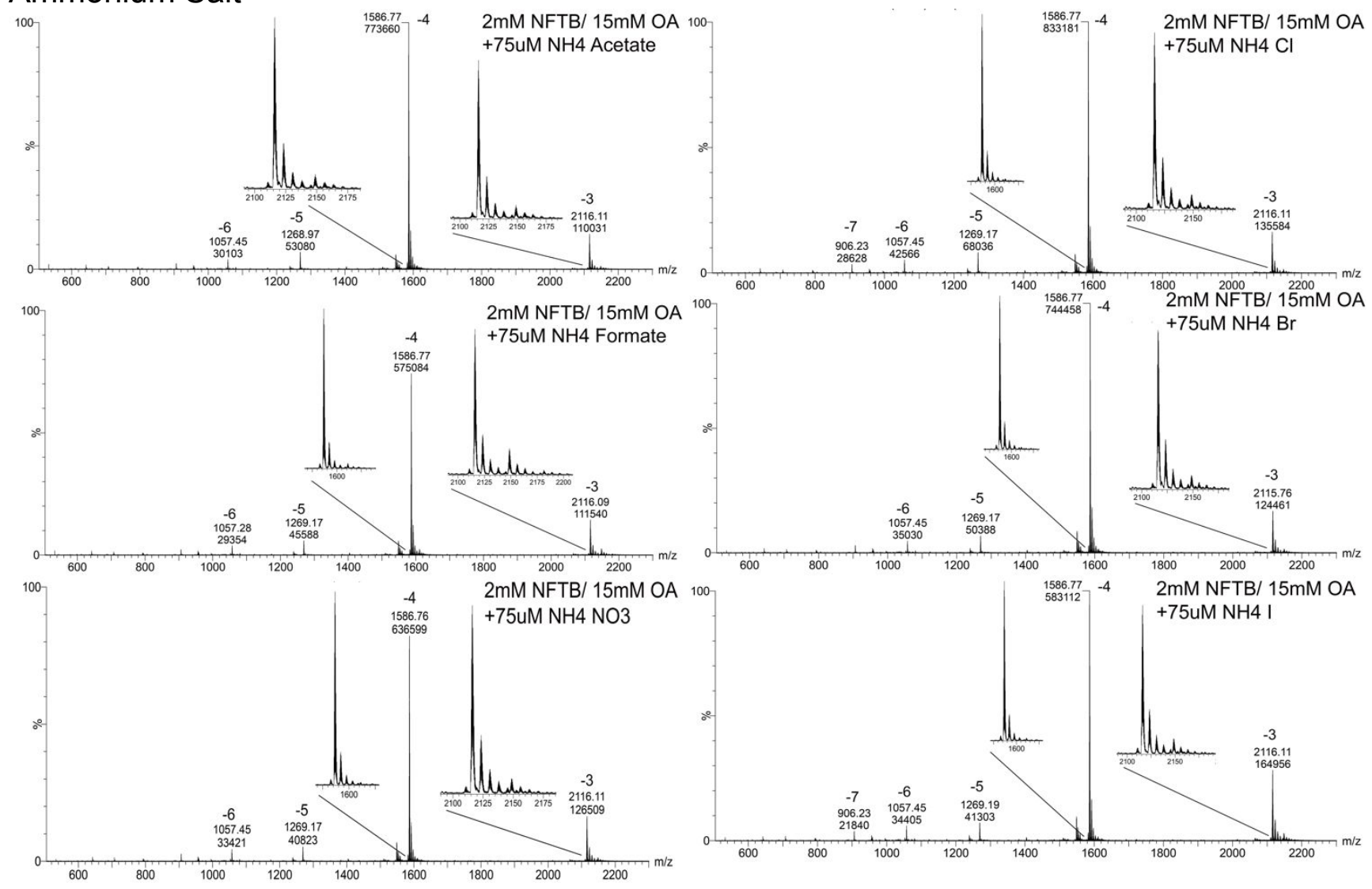

Supplemental Figure 2: $10 \mu \mathrm{M}$ Oligo 1 in 50/50 water/methanol with 2mM NFTB and increasing concentrations of OA; A: $0 \mathrm{mM}, \mathrm{B}: 5 \mathrm{mM}, \mathrm{C}: 15 \mathrm{mM}, \mathrm{D}: 25 \mathrm{mM}$ and E:50mM. 


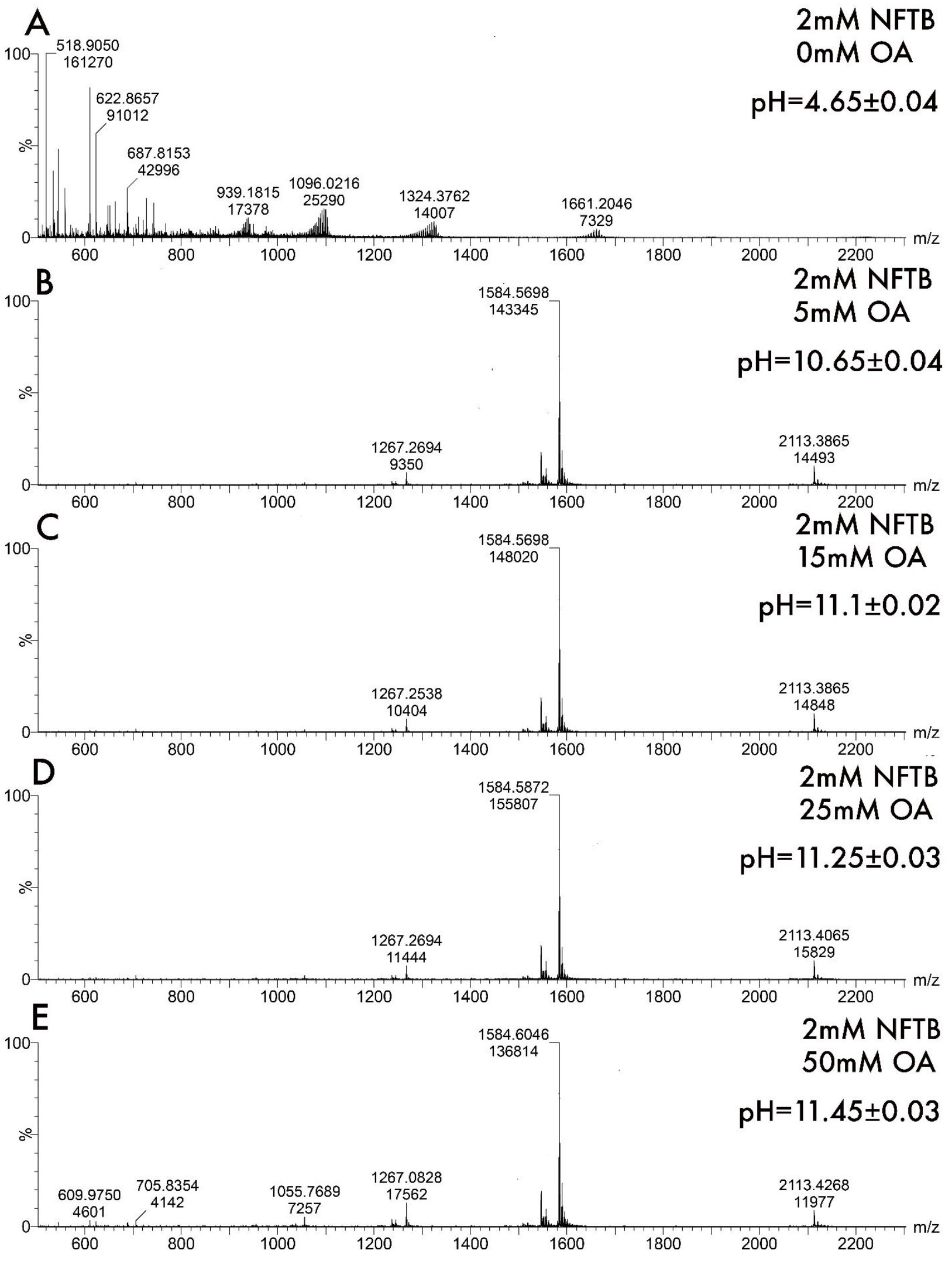


Supplementary Table 2: Collision Cross Sections calculated for HpB using the EHSS nucleic acid calibration and the protein calibration. 10uM HpB sprayed with $5 \mathrm{mM}$ Ammonium Acetate in $100 \%$ water (control), $5 \mathrm{mM}$ was optimized to obtain sufficiently high signal intensity. 10uM HpB sprayed with $2 \mathrm{mM} \mathrm{NFTB/15mM} \mathrm{OA} \mathrm{in} \mathrm{50/50}$ water/methanol. 10uM HpB sprayed with $25 \mathrm{mM} \mathrm{HFIP/15mM} \mathrm{OA} \mathrm{in} \mathrm{50/50}$ water/methanol. 10uM HpB sprayed with $2 \mathrm{mM} \mathrm{NFTB/15mM} \mathrm{OA} \mathrm{in} \mathrm{50/50}$ water/methanol $+75 \mathrm{uM} \mathrm{NH}_{4} \mathrm{SO}_{4}$.

\begin{tabular}{|c|c|c|}
\hline Charge & $\begin{array}{c}\text { CCS EHSS N2 } \\
(\Omega, \AA ̊ 2)\end{array}$ & $\begin{array}{c}\text { CCS Protein } \\
\text { Calibrants }(\Omega, \AA ̊ 2)\end{array}$ \\
\hline \multicolumn{3}{|c|}{ Ammonium Acetate } \\
\hline-4 & $1148.84 \pm 1.30$ & $1097.20 \pm 1.35$ \\
\hline-5 & $1316.32 \pm 1.57$ & $1178.19 \pm 1.56$ \\
\hline-6 & $1476.90 \pm 4.61$ & $1254.82 \pm 4.46$ \\
\hline \multicolumn{3}{|c|}{ NFTB/OA } \\
\hline-4 & $1147.97 \pm 0.76$ & $1096.30 \pm 0.78$ \\
\hline-5 & $1316.32 \pm 1.57$ & $1178.19 \pm 1.56$ \\
\hline-6 & $1478.39 \pm 6.96$ & $1256.27 \pm 6.74$ \\
\hline \multicolumn{3}{|c|}{ HFIP/OA } \\
\hline-4 & $1147 \pm 2.02$ & $1095.38 \pm 2.09$ \\
\hline-5 & $1317.08 \pm 2.69$ & $1179.08 \pm 2.69$ \\
\hline-6 & $1481.46 \pm 4.52$ & $1259.24 \pm 4.38$ \\
\hline \multicolumn{3}{|c|}{ NFTB/OA + Ammonium Sulfate } \\
\hline-4 & $1148.40 \pm 0.76$ & $1096.30 \pm 0.78$ \\
\hline-5 & $1319.00 \pm 1.54$ & $1180.87 \pm 1.54$ \\
\hline-6 & $1479.94 \pm 5.21$ & $1257.77 \pm 5.05$ \\
\hline
\end{tabular}

